\title{
Performance Analysis of Different M-ARY Modulation Techniques over wireless fading channel
}

\author{
${ }^{1}$ Vishwas Giri Goswami, ${ }^{2}$ Sandhya Sharma \\ ${ }^{1} S G V U$, Jaipur, India ${ }^{2} S G V U$, Jaipur, India
}

\begin{abstract}
Cellular communication systems are the most widely used wireless communication systems. It is our primary need today to achieve the higher data rates in limited spectrum bandwidth to improve the performance of signals. We know digital communication system outperforms analog ones in terms of noise performance and flexibility. Hence, there has been great deal of search for a digital communication system that is bandwidth efficient and has low bit error rate at a relatively low signal to noise ratio. Various digital modulation schemes are incorporated but they are not feasible or cannot fulfil actual requirement varying in different kind of environment. In this paper we provide a general theoretical approach to analyze various M-ary modulation schemes using MATLAB taking BER as measure of performance when the system is subjected to AWGN and multipath Rayleigh fading channel. Based on these performances a desirable modulation scheme is suggested that provides low BER at low received SNR, performs well in multipath \& fading conditions occupies a minimum of bandwidth and is easy \& cost effective to implement in present cellular communication.
\end{abstract}

General Terms: M-ARY Modulation, Quadrature Amplitude Modulation

Index Terms: Phase Shift Keying (PSK), Bit Error Rate (BER), Signal-to-Noise Ratio (SNR), AWGN (Additive White Gaussian Noise).

\section{Introduction}

The evolution objective of wireless cellular technology from $1 \mathrm{G}$ to $3 \mathrm{G}$ is capable of delivering high data rate signal so that it can transmit high bit rate multimedia content in cellular mobile communication [5]-[9]. There are several digital modulation schemes that have been proposed but there is a tradeoff between data rate and mismatch of the three basic parameters as phase, frequency and time between the transmitter and receiver [1].These digital modulation schemes include basic schemes such as MPSK (M-ary Phase Shift Keying), MPAM (M-ary Pulse Amplitude Modulation), MQAM (M-ary Quadrature Amplitude Modulation), MFSK (Mary Frequency Shift Keying), and GMSK (Gaussian Minimum Shift Keying). In 2G networks, GMSK modulation scheme is widely used in GSM (Global System for Mobile Communication). This modulation can only transmit data rate of 1 bit per symbol thus this kind of modulation scheme is not suitable for the next generation communication system. So, there is a need to study the performance of new modulation technique that could deliver higher data rate effectively in a multipath fading channel. M-ary modulation schemes are one of most efficient digital data transmission systems as it achieves better bandwidth efficiency than other modulation techniques and give higher data rate [11].

The objective of this paper is to review the key characteristics and performance of digital modulation schemes. Simulations are used to compare the performance and tradeoffs of $\mathrm{M}$ - ary techniques, including analysis of BER in the presence of Additive White Gaussian Noise (AWGN) and multipath fading environment. Another necessary study is to how diversity will improve the degradation of the faded signals. By applying diversity to the receiver end the version of the incoming signal which gives the best SNR can be achieved. For the wireless communication system another threat of the signals is the synchronization mismatch. The main objective is to study the degradation of the error rates due to the imperfect phase estimation of the coherently detected signals at the receiver end and to show BPSK and QPSK signals how affected due to the phase synchronization mismatch.

\section{M- Ary Modulation Techniques}

Digital baseband data may be sent by varying both the envelope and phase ( or frequency) of an RF carrier as the envelope and phase offer two degrees of freedom and modulation techniques map baseband data into four or more possible RF Carrier signals. Such modulation techniques are called M-ary modulation, since they can represent more signals than if just the amplitude or phase were varied alone [13].

In an M-ary signaling scheme, we may send one of M possible signals $\mathrm{s} 1(\mathrm{t}), \mathrm{s} 2(\mathrm{t}) \ldots \mathrm{sm}(\mathrm{t})$, during each signaling interval of duration Ts. For almost all applications, the number of possible signals $M=2 n$, where $n$ is an integer. The symbol duration $\mathrm{Ts}=\mathrm{nTb}$, where $\mathrm{Tb}$ is the bit duration. In pass-band data transmission these signals are generated by changing the amplitude, phase, frequency of a sinusoidal carrier in M discrete steps thus we have M-ary ASK, M-ary PSK and M-ary FSK digital modulation schemes [13][14][15]. Different 
bandwidth efficiency at the expense of power efficiency can be achieved using M-ary modulation schemes.

\subsection{M-ary PSK}

In M-ary PSK, the carrier phase takes on one of $M$ possible values, namely $\theta \mathrm{i}=2(\mathrm{i}-1)$, where $\mathrm{i}=1$,

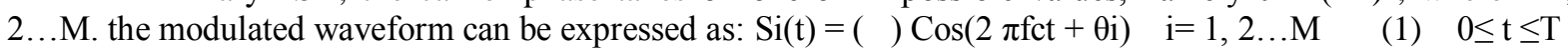
Where $\mathrm{ES}=(\log 2 \mathrm{M}) \mathrm{Eb}$ is the energy per symbol and $\mathrm{TS}=(\log 2 \mathrm{M}) \mathrm{Tb}$ is the symbol period [13]. The above equation can be rewritten in quadrature form as: $\operatorname{Si}(\mathrm{t})=\{\cos [(\mathrm{i}-1)] \Phi 1(\mathrm{t})-\sin [(\mathrm{i}-1)] \Phi 2(\mathrm{t})\}$

$\mathrm{i}=1,2 \ldots \ldots \mathrm{M}$

By choosing orthogonal basis signals $\Phi 1(t)=\sqrt{ }(2 / T s) \cos (2 \pi f c t)$ and $\Phi 2(t)=\sqrt{ }(2 / T s) \sin (2 \pi f c t)$ defined over the interval $0 \leq \mathrm{t} \leq \mathrm{Ts}$, the M-ary PSK signal set can be expressed as:

$$
\begin{aligned}
& \operatorname{SMPSK}(\mathrm{t})=\{\cos [(\mathrm{i}-1)] \Phi 1(\mathrm{t})-\sin [(\mathrm{i}-1) \\
& ] \Phi 2(\mathrm{t})\} \\
& \mathrm{i}=1,2 \ldots \ldots \mathrm{M}
\end{aligned}
$$

Since there are only two basis signals, the constellation of M-ary PSK is two dimensional. It is clear from fig.1 MPSK is a constant envelope signal when no pulse shaping is used [17].

Average symbol error probability of an M-ary PSK system is given by

$\mathrm{Pe} \leq 2 \mathrm{Q}(\sin ())$

\subsection{M-ary QAM}

By allowing the amplitude to vary with the phase, a new modulation scheme called Quadrature Amplitude Modulation (QAM) is obtained. It is such a class of non-constant envelope schemes that can achieve higher bandwidth efficiency than MPSK with the same average signal power [13].

The general form of an M-ary QAM signal can be defined as:

$\mathrm{Si}(\mathrm{t})=$ ai $\cos (2 \mathrm{fct})$

$$
\left\{\begin{array}{cl}
+ & \text { bi } \sin (2 \mathrm{fct}) \\
0 \leq \mathrm{t} \leq \mathrm{T} & \mathrm{i}=1,2 \ldots \ldots \mathrm{M}
\end{array}\right.
$$

Where Emin is the energy of the signal with the lowest amplitude and ai and bi are a pair of independent integers chosen according to the locations of the particular signal point. M-ary QAM does not have constant energy per symbol, nor does it have constant distribution between possible symbol states. It is for the reasons that particular values of $\mathrm{Si}(\mathrm{t})$ will be detected with higher probability than others [13].

If rectangular pulse shapes are assumed, the signal $\mathrm{S} i(\mathrm{t})$ may be expanded in terms a pair of basis function defined as

$$
\begin{array}{lll}
\Phi 1(\mathrm{t}) & \cos (2 \pi \mathrm{fct}) & 0 \leq \mathrm{t} \leq \mathrm{Ts} \\
= & \operatorname{sen} & \\
\Phi 2(\mathrm{t}) & \sin (2 \pi \mathrm{fct}) & 0 \leq \mathrm{t} \leq \mathrm{Ts}
\end{array}
$$

The average probability of error in an AWGN channel for M-ary QAM, using coherent detection: $\mathrm{Pe} \approx 4(1-(1 /)) \mathrm{Q}$

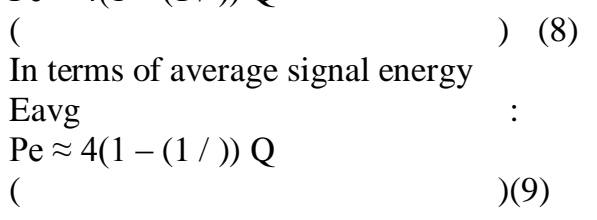

\subsection{Bit Error Rate or Probability of Bit Error}

\section{System Perfomance Measures}

BER is a performance measurement that specifies the number of bit corrupted or destroyed as they are transmitted from its source to its destination. Several factors that affect BER include bandwidth, SNR, transmission speed and transmission medium. The definition of bit error rate can be translated into a simple formula:

BER $=$ No. of Errors /Total No. of bits sent (10) 
BER can also be defined in terms of the probability of error or POE. Each different type of modulation has its own value for the error function. This is because each type of modulation performs differently in the presence of noise [17].

\subsection{SNR, signal-to-noise density ratio, (Eb is the energy per bit and No is the noise Density)}

Signal to noise ratio (SNR) is a measure of the amount of good signal divided by the amount of noise being received. In general a high Signal-to-Noise Ratio is good because it means we are getting more signal and less noise. SNR is usually measure using a logarithmic scale, meaning the SNR value is the logarithm of the actual ratio. Generally, high noise (low SNR) can lead to high BER. High BER is bad, and usually leads to observable problems with the signal. It is important to note that $\mathrm{POE}$ is proportional to $\mathrm{Eb} / \mathrm{No}$ and is a form of signal to noise ratio [15].

$\mathrm{SNR}=10 \log 10() \mathrm{dB}$

\subsection{Constellation Diagram}

Constellation diagram provides a graphical representation of the complex envelope of each possible Symbol state. The $\mathrm{x}$ - axis of the constellation diagram represents the in-phase component of the complex envelope and the $y$-axis represents the quadrature component of the complex envelope. The distance between the signals on the constellation diagram relates to how different the modulation waveform are, and how well a receiver can differentiate between all possible symbols when random noise is present.
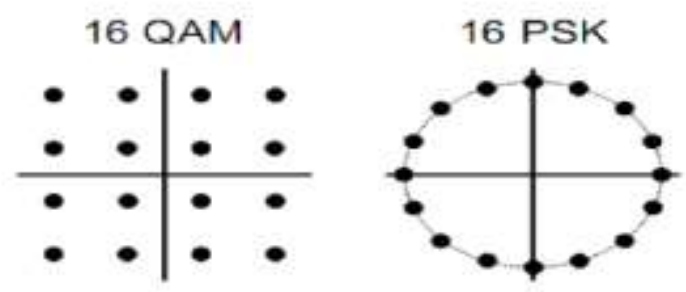

Fig.1 : Constellation Of 16QAM \& 16PSK

The Probability of bit error is proportional to the distance between the closest points in the constellation, this implies that a modulation scheme with a constellation that is densely packed is less energy efficient than a modulation scheme that has sparse constellation [13][15][16].

\subsection{AWGN (Additive white Gaussian noise)}

In communication systems, the most common type of noise added over the channel is the Additive White Gaussian Noise (AWGN). It is additive because the received signal is equal to the transmitted signal plus the noise. It is white because it has a constant power spectral density. It is Gaussian because its probability density function can be accurately modelled to signal i behave like a Gaussian distribution. It is noise because it distorts the received signal. Because the bandwidth of the ver less as compare to the bandwidth of the AWGN channel. The higher the variance of the noise, the more is the deviation of the received symbols with respect to the constellation set and, thus, the higher is the probability to demodulate a wrong symbol and make errors [15].

\subsection{Rayleigh fading}

Small-scale fading is also known as Rayleigh fading since the fluctuation of the signal envelope is rayleigh distributed when there is no predominant line of sight between the transmitter and receiver and its probability density function (pdf) is given by

Rayleigh $(\mathrm{r})=(0 \leq \mathrm{r} \leq) \quad(12)$

$$
(0 \leq \mathrm{r} \leq)
$$

Where $\sigma$ is the root mean squared value of the received signal before detection and $\sigma 2$ is the average power of the received fading signal [16].

\section{Mitigating the effect of fading by diversity}

Diversity is a commonly used technique in wireless systems to combat channel fading, due to the following reasons [25];

(1) The degradation of the transmission quality due to fading cannot simply overcome by increasing the transmitted power. Even with high transmitted power, when the channel is in deep fad, the instantaneously received SNR per bit can still be very low, is resulting a high probability of transmission error.

(2) The power of the reverse link in a wireless system is limited by the battery capacity in hand-held subscriber 
units. With diversity the required transmitted power will be reduced.

(3) Diversity limited the interferences, thus it support addition of more user and hence increases the capacity of the system.

The physical model assumes the fading to be independent from one element to the next. Each element, therefore, acts as an independent sample of the random fading process. Our goal here is to combine these independent samples to achieve the desired goal of increasing the SNR and reducing the BER. N elements in the receiving antenna array we receive $\mathrm{N}$ independent copies of the same signal. It is unlikely that all $\mathrm{N}$ elements are in a deep fade. If at least one copy has reasonable power, one should conceivably be able to adequately process the signal.

1SELECTION COMBINING: As each element is an independent sample of the fading process, the element with the greatest SNR is chosen for further processing.

2 MAXIMAL RATIO COMBING: In the above formulation of selection diversity we choose the element with the best SNR. This is clearly not the optional solution for (L-1) elements of the array are ignored. Maximal ratio combining (MRC) obtains the weights that maximize the output SNR, i.e. it's optional in terms of SNR.

\section{Simulation Results}

The graphical analysis for the performance of M -ary modulation schemes over AWGN channel are given below in Figure (2.15) to Figure (2.21).These graphs were plotted by taking SNR in dB on the abscissa and probability of error on ordinate. Figure (2.15) to figure (2.18) showed that the simulated and theoretical curves of the symbol error rate of the modulation schemes almost matched each other or the simulated curves satisfied the theoretical symbol error rates. Figure (2.19) to figure (2.21), from these figures it is observed that as the value of $\mathrm{M}$ increases the probability of error is also increases.

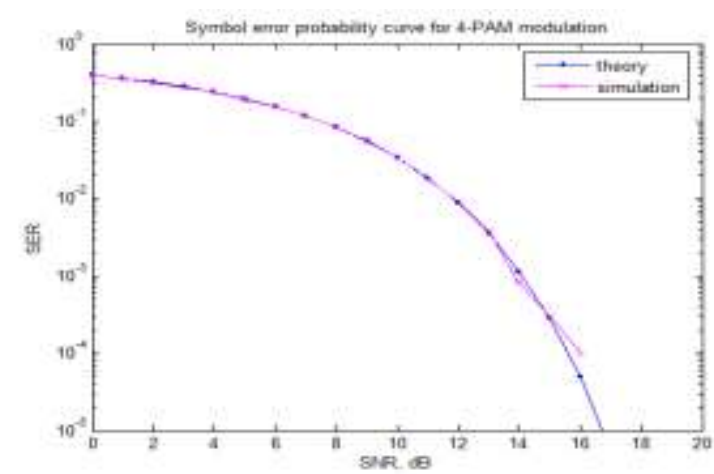

Symbol error probability (simulation and theoretical) curve of MPAM (illustrated for M=4)

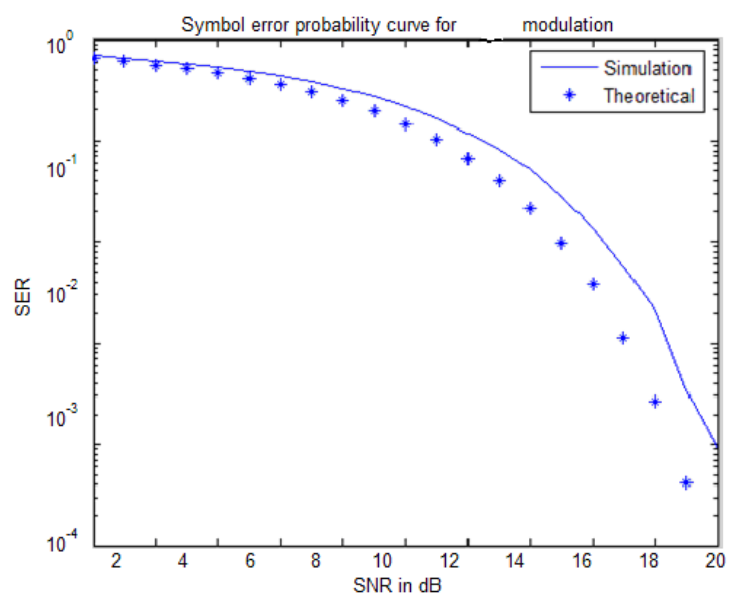

Symbol error probability (simulation and theoretical) curve of MPSK (illustrated for M=8) 


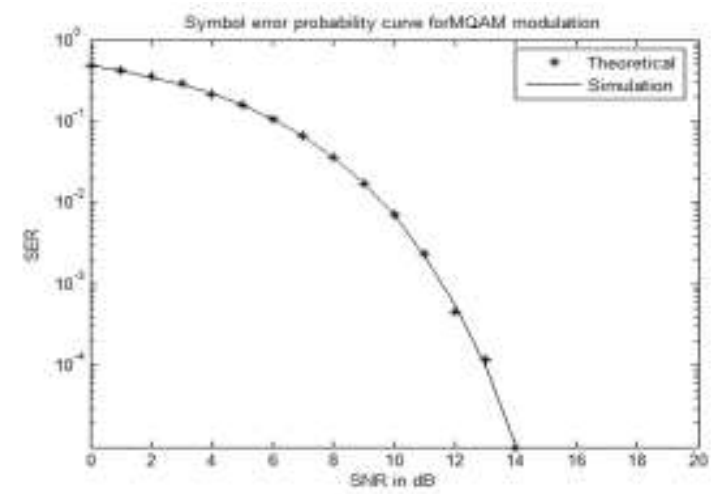

Symbol error probability (simulation and theoretical) curve of MQAM (illustrated for M=16)

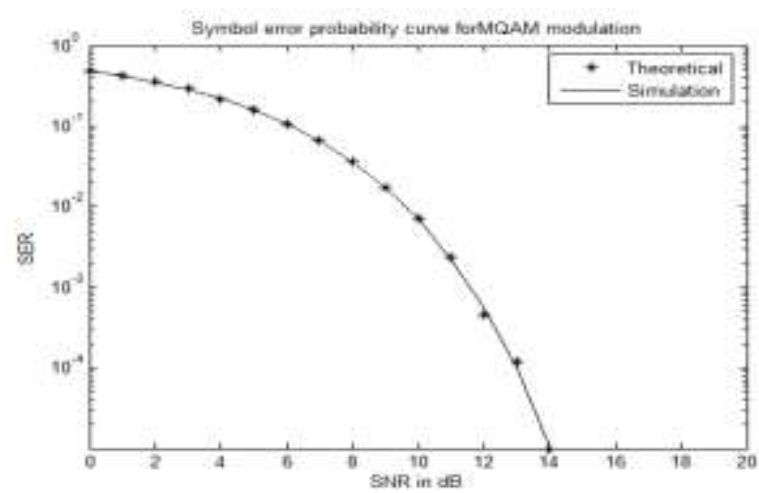

\section{Simulation Results \& Discussions}

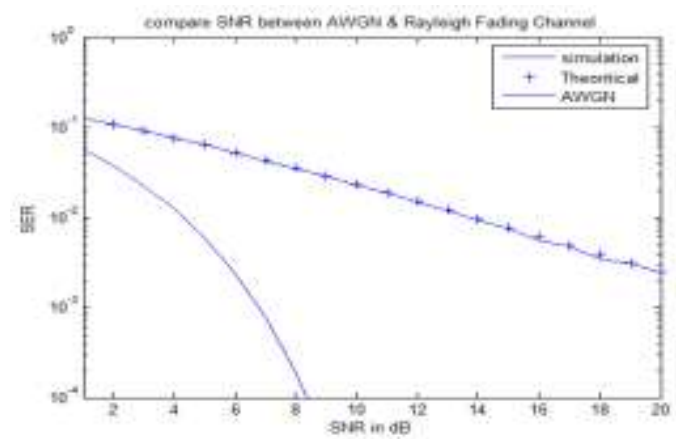

Fig. Symbol error probability (simulation and theoretical) curves of BPSK over AWGN and Rayleigh fading channel

From this fig it is observed that the amplitude fading severally degrades the transmission performance. In AWGN channel, for large value of, the probability of error decreases exponentially with respect to $y^{2}$. And in Rayleigh the probability of error decreases linearly with respect to $y$.

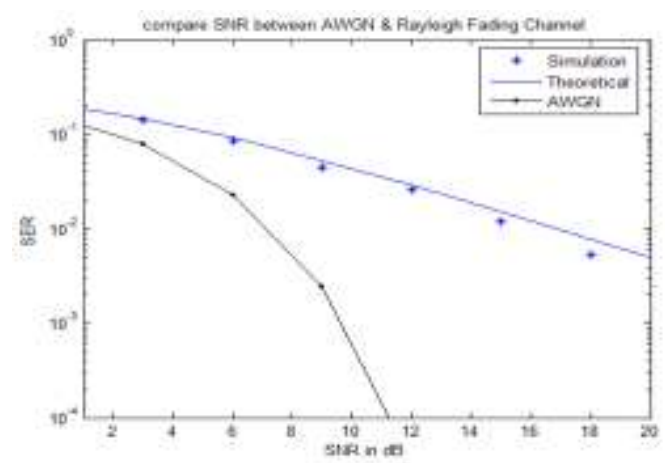

Fig. Symbol error probability (simulation and theoretical) curves of BFSK over AWGN andRayleigh fading channel 
Simulation of 'QAM over fading channel with diversity order 1 to 20 '

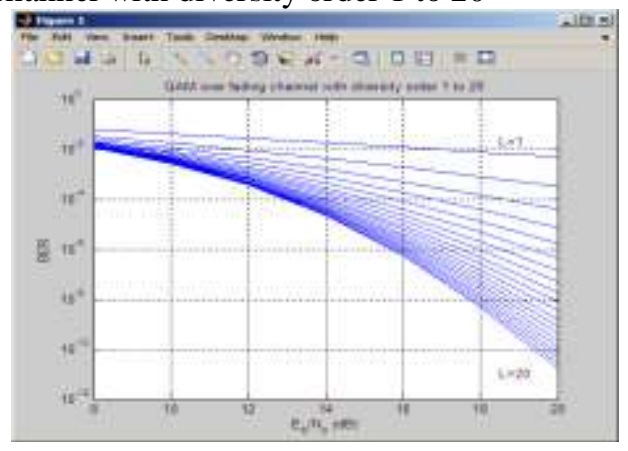

The BER for BPSK modulation in a Rayleigh fading channel with selection diversity

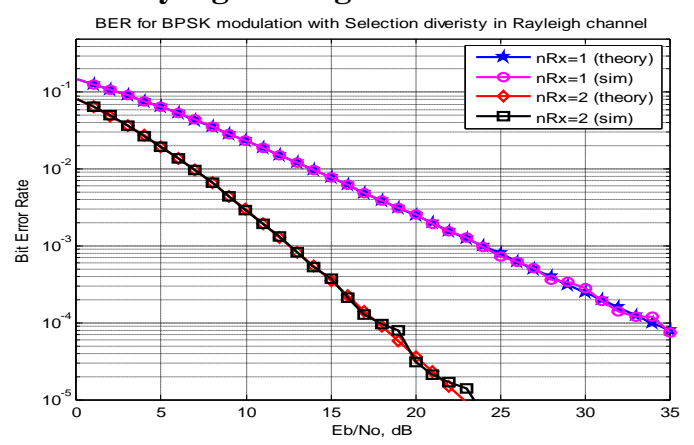

SNR improvement by selection combing

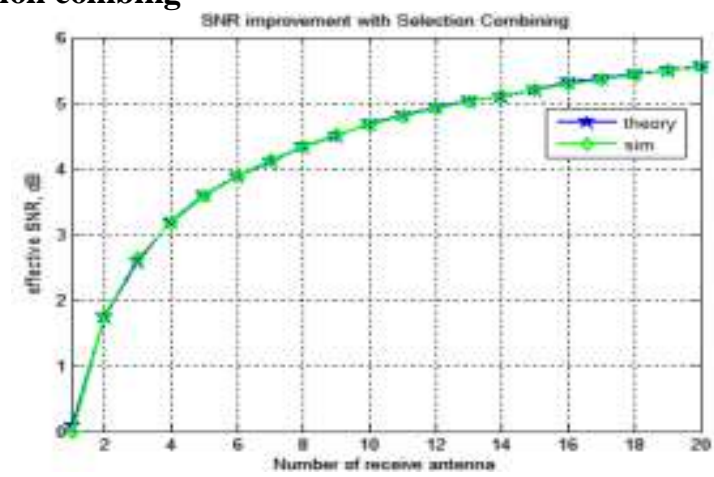

With two receive antennas the effective bit energy to noise ratio is 1.5 times

With three receive antennas, the effective bit energy to noise ratio is 1.833 times,

With four receive antennas, the effective bit energy to noise ratio is 2 times and so on.

If you recall the results from the AWGN with receive diversity case,

Effective bit energy to noise ratio in a $\mathrm{N}$ receive antenna case is $\mathrm{N}$ times the bit energy to noise ratio for single antenna case.

With selection diversity we are seeing that the effective SNR improvement is not a linear improvement with increasing the number of receive antennas. The returns diminish.

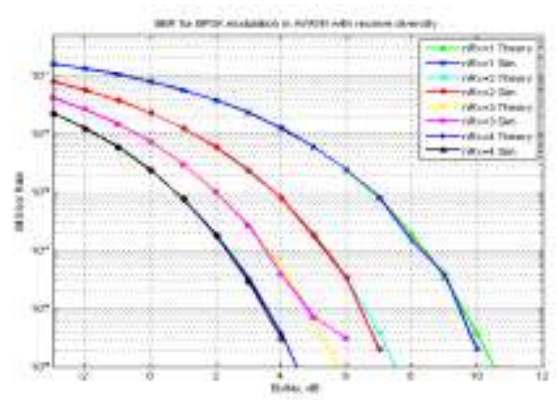

for BPSK modulation in AWGN with receive diversity' 


\section{Conclusions}

The focus of this paper is on the mathematical analysis of the M-ary modulation schemes over AWGN channel and different Fading channel environment. And focuses on performance of signals over different diversity techniques.

More specifically, it focuses on the performance analysis of the coherent phase modulation with imperfect phase estimation.

This paper begins with an elaborate discussion of the M-ary modulation schemes as, M- ary Amplitude modulation, M-ary Phase shift keying, M-ary Frequency shift keying, M-ary Quadrature Amplitude modulation, Differential M-ary phase shift keying with their Geographic and Baseband representation. It mainly contains the analysis of the probability of error of the M-ary signals over Additive White Gaussian Noise (AWGN) channel. From the simulation curves and the mathematical analysis of the signals it is observed that as the number of signals or number of $\mathrm{M}$ increases $(\mathrm{M} \geq 0,1,2, \ldots, \mathrm{M})$ the error probability or more clearly the probability of Symbol error rate is increased. It was also observed that the Binary Frequency Shift Keying is giving the least probability of error over AWGN channel. All this analysis is very much important for the basic analysis of any communication system, as the digital M-ary modulation offers much more advantages than other modulation schemes.

The analysis of Binary Phase Shift Keying (BPSK) and Binary Frequency Shift Keying (BFSK) is done over Rayleigh. The probability of error is increased in case of BFSK signal in Fading channel, and it showed worst error performance than the BPSK signal, which is the reveres case if consider the performance over the AWGN channel.

\section{Acknowledgement}

We would like to cordial thanks to Dean, HOD of dept. of Electronics and Communication engineering and faculties of Electronics and Communication engineering. The study work is supported by communication laboratory and its annual allowance of College of technology and engineering, GYAN VIHAR UNIVERSITY, Jaipur (Raj.) India.

[1] Simon Haykin,'Digital Communication" Edition, 2006

[2] Http://En.Wikipedia.Org/Wiki

[3] I. S. Gradshteyn And I. M. Ryzhik, Table of Integrals, Series and Products. San Diego, Fifth Edition, Ca: Academic, 1994.

[4] J. Sun And I. Reed, "Linear Diversity Analyses For M- Psk In Rician Fading Channels," IEEE Trans. Commun.,Vol. 51, No. 11, Pp. 1749- 1753, Nov. 2003.

[5] Y. Rosmansyah, P. Sweeney, R. Tafazolli, “Air-Interface Techniques For Achieving High Data Rates For Umts”, IEEE 3g Mobile Communication Technologies, Conference Publication No. 477, Pp. 368-372, 26-28 March 2001.

[6] A.S. Madhukumar, Francois Chin, "An Efficient Method For High-Rate Data Transmission Using Residue Number System Based Ds-Cdma", IEEE.

[7] Min-Yan Song, Yang Xiao, Joachim Habermann, “High Data Rate Wireless System”, IEEE, Pp. 1344-1350.

[8] Haifeng Wang, Zhenhong Li, "Novel Soft-Bit Demodulator with Multi-Dimensional Projection for High-Order Modulation", IEEE, Pp. 2051-2054, 2002.

[9] Troels Emil Kolding, Klaus Ingemann Pedersen, Jeroen Wigard, Frank Frederiksen, Preben Elgaard. Mogensen, "High Speed Downlink Packet Access (Hsdpa): W-Cdma Evolution”, IEEE Vehicular Technology Society News, February, 2003.

[10] E. Hossain, T. Issariyakul, "Performance Bound Of Dynamic Forward Link Adaptation In Cellular W-Cdma Networks Using High Order Modulation and Multicode Formats”, IEEE Electronics Letters, Vol.40, No. 2, January 2004.

[11] Aun Ali Tahir Feng Zhao "Performance Analysis on Modulation Techniques of W-Cdma in Multipath Fading Channel" Electrical Engineering Blekinge Institute Of Technology January 2009.

[12] Feher, K.: Wireless digital communications, entice-Hall, New Jersey, 1995.

[13] Rappaport, T.S.: Wireless communications: Principles and Practices, Prentice-Hall, New Jersey, 1996.

[14] S. Haykin and M. Moher, Modern Wireless Communications, Pearson Prentice Hall, 2005

[15] A F. Molisch, Wireless Communications, John Wiley \& Sons, 2005

[16] A. Goldsmith, Wireless Communications, Cambridge University Press, 2005

[17] J. G. Proakis, Digital Communications, $4^{\text {th }}$ Edition, McGraw Hill, 2000 